\title{
TV/Series
}

1 | 2012

Les Séries télévisées américaines contemporaines :

entre la fiction, les faits, et le réel

\section{Desperate Housewives - fiction de toutes les fictions}

\section{Renan Cros}

\section{(2) OpenEdition}

Journals

Édition électronique

URL : http://journals.openedition.org/tvseries/1563

DOI : 10.4000/tvseries.1563

ISSN : 2266-0909

Éditeur

GRIC - Groupe de recherche Identités et Cultures

Référence électronique

Renan Cros, "Desperate Housewives - fiction de toutes les fictions », TV/Series [En ligne], 1 | 2012, mis en ligne le 15 mai 2012, consulté le 04 mai 2019. URL : http://journals.openedition.org/tvseries/1563 ; DOI : 10.4000/tvseries. 1563

\section{c.) (i)}

TV/Series est mis à disposition selon les termes de la licence Creative Commons Attribution - Pas d'Utilisation Commerciale - Pas de Modification 4.0 International. 


\section{Desperate Housewives - fiction de toutes les fictions}

Fiction de toutes les fictions, Desperate Housewives, entre ironie et empathie, accumule et désarticule les codes du soap opera pour mettre sur l'écran l'imaginaire d'une nation. À partir d'un récit balisé, la série fait de la répétition un mode de lecture. Répétition des situations mais aussi répétition de fictions préexistantes, Desperate Housewives fait du feuilleton l'art de la répétition en célébrant la culture populaire américaine.

$D$

esperate Housewives se terminera en mai 2012 après 8 saisons. A l'heure où cette huitième saison opère une boucle narrative passionnante sur les origines de la série, il est utile de se pencher sur le fonctionnement de cette œuvre maîtresse du renouveau de la série télévisée contemporaine américaine, aussi populaire que conceptuelle. Cependant, un certain désamour critique et une chute d'audience ont entaché la grandeur du projet de Marc Cherry. Après une première saison à succès, la série a perdu de ses fidèles au fil des saisons. Pourtant, c'est sur la durée que se savoure la série. Exemple parfaite de la "soapisation» grandissante des séries télévisées américaines contemporaine, Desperate Housewives oblige le spectateur à s'accrocher à son téléviseur quoiqu'il advienne sur l'écran. Ainsi, comment rentrer dans une fiction qui ne se termine pas? La question paraît abrupte et nécessite que l'on emprunte quelques chemins détournés.

Une caractérisation précise des systèmes sériels et feuilletonesques s'opère à travers le suivi du spectateur entre la variation et la répétition. Lors d'une pure structure sérielle, comme la sitcom, le spectateur s'intègre au cœur d'une boucle, d'un circuit narratif imagé dont il peut à tout moment s'extraire. Le suivi provient de sa capacité et de son plaisir, à s'approprier cette mécanique sérielle². Le spectateur s'implique ainsi dans une mécanique narrative à courte durée. Le récit prend une forme minimale et s'efface sous la puissante évocation du quotidien. La structure feuilletonesque dès lors soulève un problème de caractérisation.

Le principe même du «feuilleton » est d'impliquer à long terme le spectateur au cœur de la mécanique narrative. On pourrait

\footnotetext{
1 L'acceptation de cette boucle diégétique passe notamment par un fort processus de reconnaissance et d'identification, à travers l'équivalence des quotidiens.
} 
imager la structure feuilletonesque comme un circuit distendu qui tendrait, peu à peu, au fil des épisodes, à se resserrer autour du spectateur, pour finalement, au tout dernier instant, se dilater à nouveau. C'est l'utilisation, par exemple, d'un cliffangher, figure narrative qui consiste à mettre en suspend le récit pour mieux le faire repartir ensuite (La mort probable d'un personnage, une révélation suprise, etc...) Le feuilleton ne fait que fantasmer une résolution finale, qu'il n'est jamais tenu de fournir. La clôture d'une portion de circuit en révèle une nouvelle encore plus vaste, que le spectateur doit parcourir. La dernière séquence de la saison 2, où, Orson Hodge après avoir renversé Mike Defilno, rentre chez Bree Van De Kamp, oblige le spectateur à prolonger son suivi pour découvrir qui est réellement ce personnage. Il est donc face à un défilement sans fin, face à une mécanique qu'il ne peut caractériser puisque constamment en mouvement. Cette structure nous invite donc à questionner le rapport qui peut exister entre le spectateur et cette fiction «instable » et finalement inconnue, puisque jamais totalement cohérente ${ }^{2}$.

La construction d'une fiction passe par la stabilité du spectateur et, de manière presque implicite, son contentement. La fiction doit installer un rapport de confiance qui semble de prime abord impossible dans le feuilleton: programmant toujours une frustration imminente, elle défie sans cesse le principe de savoir propre au spectateur. Il est toujours dans une position d'attente, si ce n'est pire, de suspense 3 , que seul le temps viendra résoudre, lors d'un prochain épisode, riche lui aussi en divers effets de frustration.

La question n'est donc tant pas tant la fin de la fiction que sa capacité à nous inclure en elle. Par quels moyens, quels procédés, outre justement cette frustration narrative, ce type de système fictionnel conserve-t-il une cohérence, une adhésion du spectateur ? La question de l'adhésion fictionnelle est primordiale puisqu'elle est essentielle à la perduration de cette fiction. Il faut donc trouver le lien qui permet de réunir ensemble toutes ces formes de ruptures que provoque la fiction feuilletonante. Le récit étant lui-même fractionné, il ne peut servir de lien efficient et durable. Le feuilleton doit donc se départir d'une pure logique d'événements pour se construire selon des axes d'images et de sens. La série télévisée contemporaine américaine repense ces liens. En s'interrogeant sur le statut de l'image au cœur de ce mouvement

\footnotetext{
${ }^{3}$ La notion de suspense implique une prescience du spectateur sur le personnage. Ce type de "non fermeture » d'épisode est très fréquent dans la série télévisée contemporaine. Le spectateur percoit un élément décisif dont le personnage n'aura conscience que lors du prochain épisode. L'effet est saisissant parce qu'il impose un rôle réactif au spectateur propre à l'identification : il est le garant d'un savoir qu'il rêve de transmettre aux personnages derrière l'écran. Ce type de structure induit un effet de "non communicabilité » qui, là encore, par le passage du temps, crée une situation de défiance face à la fiction.
} 
permanent, en construisant un spectateur dynamique, elle produit des séries éminemment complexes et puissantes. Mais surtout, la série télévisée contemporaine se met à repenser la fiction à l'échelle d'un homme, d'une société et d'une nation. Elle dessine de nouveaux chemins en empruntant avec un délectable art de la récup les chemins balisés des fictions matrices, connues et reconnues. Ici, l'art de la citation laisse place à une véritable esthétique fictionnelle, où le mot et l'image s'imbriquent dans un mouvement novateur qui emporte un spectateur médusé face à cet écran sur lequel défilent le nouveau et l'ancien à toute allure.

Quoi de plus trompeur que le quotidien ? En prenant appui sur l'évocation du quotidien de cinq américaines moyennes, dans une banlieue aisée, Desperate Housewives joue une carte trompeuse. Le quotidien est le lieu de toutes les promesses fictionnelles. Il est le temps qui passe, et si les images défilent alors la fiction peut se dérouler. A Wisteria Lane, quartier résidentiel de Fairview, tout semble se passer en silence. L'irruption d'un drame va mettre en branle le système fictionnel et nous allons suivre les aventures de Bree, Lynette, Susan, Gaby et Edie au cœur de cette petite banlieue paisible. Certes, si l'on sait depuis quelques fictions matrices comme Dallas ou Peyton Place que le quotidien cache souvent une sourde tempête, on n'était sûrement pas préparé à une telle déferlante fictionnelle. La série produit un art du rebondissement permanent. Mais cet art n'est que l'exemple d'une logique fictionnelle dite «de l'accumulation ». Desperate Housewives construit son récit comme un cadre au sein duquel viennent se répéter et se révéler les structures et les gestes de fictions reconnus Le spectateur s'insère alors dans un grand livre d'images, dans la fiction de toutes les fictions.

\section{De l'extérieur vers l'intérieur}

Si le quotidien est un terrain propice pour construire durablement une fiction, la série télévisée ne peut se contenter d'une simple évocation réaliste. Comprendre la place et le fonctionnement du regard qui dirige permet de comprendre les intentionnalités de la fiction mise en place pour faire du quotidien un récit. Le quotidien de Wisteria Lane apparait alors comme un labyrinthe tortueux où les personnages se croisent sous le regard distancié d'un personnage off. Alors, les allées propres, les gazons tondus et les façades immaculées deviennent la cage d'un vivarium étrange. Guidé par cette voix entomologique, le spectateur est invité à pénétrer au cœur d'une fiction analytique.

Etonnamment, la série qui s'affirme comme le feuilleton contemporain par excellence, entretient des rapports étroits avec un système sériel propre à la sitcom. En effet, dans Desperate Housewives, la fiction s'articule autour des quatre personnages 
principaux, chacune ayant une galaxie de personnages propre. Quatre quotidiens semblent se juxtaposer. La série suit bien, en effet, le quotidien de Susan, Bree, Gaby et Lynette : on pénètre dans la vie de chacune pour attraper des courts instants de leur vie. On serait donc face à une structure plus proche du sériel où le quotidien est variation au sein du même. Ainsi, on voit bien comment, de prime abord, un épisode se construit autour d'une addition de séquences, qui amène le spectateur à jongler entre les différents quotidiens. On passe du débordement de Lynette aux gaffes de Susan, du contrôle hystérique de Bree aux problèmes conjugaux de Gaby. La structure du gag induit, de plus, une unité de séquence, qui renie tout effet de circuit prolongé, propre au feuilleton. Dans le gag, la séquence est unité puisqu'elle implique un développement corporel, un dérèglement unique, construit dans une temporalité précise. Le gag fonctionne de manière autonome et renvoie le spectateur à un présent de l'intrigue. Ainsi, on voit bien, comment, dans la série, on trouve des séquences gag-unité qui tendent à rappeler une structure proche de la sitcom. Susan se retrouve nue sur son perron. Comment va-t-elle faire pour rentrer chez elle ? La séquence se développe autour de cette question et implique bien un dérèglement corporel unique et surprenant. L'arrivée de Mike, le prince charmant sous forme de plombier, vient renforcer l'aspect comique du dérèglement.

Mais justement, cette intrusion du voisin est symptomatique de la construction hybride et novatrice de la série. Le gag est soudainement détourné par la présence d'un tiers, qui vient rappeler les enjeux feuilletonesques et fictionnels. La nudité de Susan est d'autant plus gênante que quelques épisodes plus tôt, Susan avoue son intérêt pour le plombier. Le gag s'insère donc ici dans un suivi narratif, un développement fictionnel propre au suivi du feuilleton. Ici, on retrouve le cas particulier de la love story contrariée, structure fictionnelle maintes et maintes fois reprise. Mais, au delà de ces aléas du sentiment, l'autre, le voisin est bien le garant d'un passé fictionnel. Il est le lien fictionnel qui permet de construire un récit unique. Le premier épisode dessine cette construction rhizomatique. On découvre, en un rapide montage de séquences, le portrait en forme de quotidien de nos quatre héroïnes principales ${ }^{4}$. Ces courtes séquences forment une unité, et leur juxtaposition induirait une construction parallèle.

Mais soudain, les quatre jeunes femmes se retrouvent autour d'une table, pour évoquer celle qui manque. La présence de l'autre amène le rapprochement de ces quotidiens. La grande idée de Desperate Housewives c'est de faire du voisinage le garant du feuilletonesque. Ainsi, l'autre est toujours celui qui rassemble, réunit.

4 Edie Britt, la vamp de quartier, ne s'insère vraiment dans la série que quelques épisodes plus tard. Pas véritablement un personnage principal, elle n'en devient pas moins, au fil des épisodes, une desperate housewife. 
Dans le soap classique, le quotidien est feuilleton puisqu'il n'est que relation complexe à l'autre. Le soap classique ne fait que créer des nœuds entre les personnages. On voit bien, comment, dans ce type de récit, la fiction n'est faite que de conflits, de rencontres dramatiques. Le quotidien n'a finalement pas tant sa place, puisqu'il s'efface sous le joug du nœud fictionnel, sans cesse renouvelé. La spécificité de Desperate Housewives c'est finalement de laisser une certaine autonomie à ses personnages. La fiction respire par le quotidien, elle se permet le luxe de ne «rien" raconter si ce n'est le déroulement répétitif des jours et des actions à Wisteria Lane

La série obéit donc à une double dynamique fictionnelle : le personnage s'inclut dans deux fictions, le quotidien sériel et le feuilleton, à travers sa relation à l'autre. On bascule très vite du sériel au feuilletonesque : les personnages se réunissent pour soudainement s'impliquer ensemble dans une même fiction. Il n'y a donc pas tant coexistence des fictions, qu'une seule et même fiction aux ramifications denses et complexes. C'est la logique du fil conducteur. La singularité de Desperate Housewives se situe dans cette équivalence entre le feuilleton pure (rebondissements et récits au long court) et le quotidien (juxtaposition des petites histoires liées à chacun des personnages). Contrairement aux structures en arc utilisée dans bon nombre de séries aujourd'hui, où l'on étire sur quelques épisodes le feuilleton, Desperate Housewives a été l'une des premières séries à entremêler de manière aussi dense les différentes strates de récits, rendant quasi impossible leurs distinctions. Cela oblige le spectateur à être attentif à tout ce qui se passe puisque feuilleton et quotidien sont mêlés. Lors des six saisons, nos cinq héroïnes sont amenées à élucider, ensemble, le mystère de l'autre ${ }^{5}$, mystère qui tend, bien souvent, à les impliquer elles-mêmes. Les différents quotidiens se mélangent donc soudainement dans un suivi feuilletonesque. A la fin du premier épisode, les quatre jeunes femmes se retrouvent sur le perron de la maison de Mary-Alice, leur amie défunte. On sait, à travers les divers flash-backs, qu'elles se réunissaient toutes pour parler ensemble de leur quotidien. On notera ici combien le démarrage feuilletonesque par cette réunion des identités, des quotidiens est inscrit dans une ritualité. On se demande comment ce rituel peut perdurer. Les quatre jeunes femmes sont sur le trottoir, elles vident les affaires de leur amie défunte. La découverte d'une lettre de chantage dans une poche de

\footnotetext{
5 Dans la saison 1, elles sont amenées à élucider le suicide de Mary-Alice, leur amie. La saison deux, tout en continuant à démêler le fil conducteur de cette première saison, voit l'arrivée de nouveaux voisin, les Applewhite. L'autre est renouveau du mystère, puisqu'elles seront amenées à enquêter sur les agissements de cette famille. Lors de la saison 3 , c'est la rencontre avec Orson Hodge, le nouveau mari de Bree qui amènera les quatre héroïnes à se réunir à nouveau. L'autre est véritablement le garant d'un maintien fictionnel. L'étranger est promesse d'un nouveau départ feuilletonesque comme chacune des six saisons le prouvent.
} 
veste va transformer cette ritualité du «gossip» en une véritable machine feuilletonesque. C'est le mystère d'un proche qui les réunit. La caméra entame un long travelling arrière, en plongée, et isole alors ce petit groupe de femmes au cœur d'un plan d'ensemble de cette banlieue paisible. Le plan, à lui seul, résume de manière saisissante la série. Ces femmes forment un noyau au cœur de cette machine autonome du quotidien. Les gens autour d'elles s'agitent mais, elles, elles forment un tout, une unité dirigée, qui enclenche la fiction. Dans ce plan, on retrouve bien les deux axes de la série : autour d'elles s'agite le monde, leur quotidien, mais elles, au centre, sont aux prises avec des relents dramatiques, des interrogations mystérieuses qui induisent alors un développement feuilletonesque.

En jonglant avec virtuosité sur ces deux pendants, la série crée donc une fiction chorale dirigée, où le rebondissement n'est jamais loin d'un détail comique, où la tension s'accompagne toujours d'une échappatoire par le quotidien. La série n'établit d'ailleurs pas véritablement une hiérarchie. Le destin personnel des quatre jeunes femmes peut soudain prendre la place du fil conducteur feuilletonesque. On voit notamment, à travers les saisons deux et trois, combien le personnage de Bree voit son quotidien devenir le terrain des mystères et des crises propres au feuilleton. Le suivi est donc tout autant lié à une identification des personnages, par leur quotidien, qu'au besoin de résolution du mystère générateur. Pour autant, la série obéit aussi à un principe liant fort : l'art de la narration, une narration étrange et puissante qui invite le spectateur, au cœur de ce quotidien feuilletonesque, à s'interroger sur le sens de ce qu'il regarde.

Quelles narrations pour quelles fictions? Si dans le soap classique, la narration s'efface derrière le pur récit ${ }^{6}$, avec Desperate Housewives, le soap mute et devient narration. On entend par " narration » l’idée que soudainement le récit est pris en charge par une tierce personne, produisant alors une situation de communication. Le soap n'apparaît donc plus tant comme l'enregistrement d'un monde que la transmission de celui-ci. Le fil de la voix guide le spectateur. Le premier épisode identifie tout de suite cette voix. Par un effet de plongée7, nous pénétrons dans Wisteria Lane et dans un élégant travelling nous parcourons les palissades immaculées pour nous arrêter devant une petite allée. Au bout, une immense maison, symbole d'une «American way of life » accompli. La porte s'ouvre, une femme

\footnotetext{
${ }^{6}$ Dans le soap, il n'y a justement pas véritablement de point de vue précis, d'ancrage narratif puisque le soap s'articule simplement sur les rebondissements permanents d'un récit. On voit bien comment, dans le soap du type Dallas, Dynastie ou Les Feux de l'amour, le récit prime sur une possible construction narrative. Les personnages sont perçus comme des existences actantielles, des purs objets de récit.

$7 \mathrm{La}$ plongée-travelling avant/arrière est un effet récurrent du récit. Ce mouvement d'appareil implique une pénétration, un franchissement de distances, symptomatique du mouvement de la série. Le regard semble soudain se focaliser.
} 
sort. Elle se présente tout de suite, par la voix. Cette entrée de la voix est saisissante puisqu'elle est directement adressée au spectateur. La présence du «vous» implique une véritable situation de communication propre à la transmission. Pour autant, dès le départ, cette transmission semble biaisée. La voix paraît décalée par rapport à l'image. La voix projette un futur que l'image n'actualise pas. Il y a donc une bascule temporelle puisque la voix nous parle une semaine après les faits. Le corps présent est donc un corps fantomatique, un corps passé. La voix annonce un fait marquant qui va se dérouler sous nos yeux. Avec elle, nous remontons le cours de cette journée décisive, jusqu'à l'instant fatal du suicide. Nous assistons, face écran, au suicide de cette paisible femme au foyer. Toute cette séquence produit un effet étrange, tant la voix semble être en permanence une redite de l'image. La voix décrit un passé pour le revivre. Mais pour autant, aucun affect. Le suicide apparait glacé, comme figé dans un quotidien répétitif. Le corps s'effondre mais la voix perdure.

Il est essentiel, ici, de faire un point sur les narrations postmortem. La plus célèbre et célébrée, bien sûr, c'est celle de Sunset Boulevard (B.Wilder, 1959). Le cadavre flottant dans la piscine nous raconte son devenir tragique. Mais le film de Wilder joue avec les codes du film noir. La série est plus proche, ici, d'un film plus contemporain : American Beauty de Sam Mendes. L'ouverture des deux œuvres semble très proche tout en étant fondamentalement opposée. On retrouve dans les deux cas ce plan plongé sur une banlieue aisée américaine. Apparition de la voix off, en surplomb. Là aussi, la voix implique une narration tragique : le personnage prédit sa mort future. Mais, contrairement à Sunset Boulevard ou American Beauty, Desperate Housewives n'est pas un long flash-back mortifère. Le personnage applique sa sentence dans les 5 premières minutes de la série. La voix off n'est donc pas fantôme en devenir (Joe et Lester dans les deux œuvres ont un regard décalé sur eux-mêmes). Mary-Alice est un fantôme. La séquence liminaire de la série n'est qu'une mise au point, un véritable flash-back ${ }^{8}$.

On se rend bien compte, par les effets de montage, que nous sommes dans un espace représenté. Les séquences rapides rendent comptent d'un quotidien répétitif. Mary-Alice commente cette journée, en énonçant ses tâches quotidiennes. Là encore, la voix et l'image se fondent dans un même sens. Le "telling » se superpose au « showing ", formant un tout imagé trépidant. Mais que faire d'un corps mort? L'effondrement de notre "narrateur» pose alors le problème de la focalisation. On admet parfaitement que le personnage, même mort, ait pleinement conscience de son corps quotidien. Pour autant, la voix est éminemment plus complexe. Le corps mort semble

${ }^{8}$ La série ne commence réellement qu'une semaine plus tard, comme le prouve la suite de l'épisode. 
amener un flottement de l'âme. Soudain, nous franchissons les murs de la maison, pour nous retrouver chez la voisine. La voix fait lien en induisant une simultanéité, entre le coup de feu et l'image. Mary-Alice s'immisce dans un autre quotidien, lié au sien. Pour autant, et c'est là, que la voix prend tout son envol, Mary-Alice pénètre dans l'intimité de ce personnage. Elle épouse, par la voix, sa subjectivité. On est donc, là, face à une nouvelle focalisation. Les personnages sur l'image sont vus de l'extérieur mais la voix nous entraîne, par le biais d'une tierce personne, au cœur de leur intimité, de leur subjectivité9. Pour autant, Mary Alice reste toujours une entité à part entière. On est donc face ici à ce que l'on nomme un discours indirect libre. Le personnage est à la fois vu «avec » et par derrière. La voix de la morte peut, à tout moment, nous faire pénétrer dans l'intériorité d'un personnage qui, par l'image, s'affirmait comme une entité extérieure. La fin de cette séquence liminaire, où Martha Huber passe soudain du chagrin à l'opportunisme est éloquente. La voix nous entraîne dans les fluctuations psychologiques du personnage, avec en prime un commentaire personnel.

Ainsi, le personnage, en lui-même, ne prend jamais la parole. Il est toujours soumis à la vision omnisciente et omniprésente du fantôme. C'est à travers lui que nous pénétrons ces personnages. Au fil de la série, Mary-Alice, garante d'un secret par lequel elle attise notre curiosité, va donc porter ce regard à la fois distant et intime sur tous les personnages. Le personnage est donc tantôt intériorité, tantôt pure extériorité. Seul le regard-voix de Mary Alice peut nous renseigner. Nous sommes soumis à elle. Ainsi, lors de la fin de la saison deux, lorsqu'Orson, un personnage annexe, écrase Mike, le plombier, Mary Alice se garde bien de nous expliquer le pourquoi de cet acte. Par ce pouvoir de pénétration, elle est le garant de toutes les zones d'ombre, de tous les secrets des personnages, qui font le sel de la série. Cette dynamique entre intériorité et extériorité développe toute la grande thématique de l'apparent calme propre à la série. Elle s'articule sur un franchissement des apparences. Qu'y a-t-il derrières les grandes façades de ces maisons parfaites ? La série nous invite à franchir le seuil de la maison et à oser s'aventurer dans le territoire effrayant de l'horreur domestique. Guidés par cette voix, qui transcende les corps et les esprits, nous franchissons les murs et nous pénétrons dans l'intimité des personnages.

Mais attention, ce mouvement de dévoilement s'accompagne toujours d'une zone d'ombre. Les personnages sont toujours une entité mystérieuse parce qu'ils sont imprévisibles. Le regard de Mary Alice n'est jamais totalement omniscient. Le discours indirect libre implique une forme de décalage propre à la critique. Le spectateur se retrouve dans un flottement permanent: entraîné par cette voix toute

${ }^{9}$ La voix traduit les émotions, les pensées et les questions de ces personnages. 
puissante ${ }^{10}$, il pénètre dans un monde où "surface et profondeur se rejoignent, pour devenir indiscernables ${ }^{11}$. En effet, il y a dans la série une étrange dimension abyssale, où tout semble à la fois extrêmement lisible et en même temps d'une complexité extrême. En ne laissant jamais la parole aux autres personnages, Mary Alice induit un véritable mystère de l'autre, dont elle est le seul garant. La fin de la saison 1 est un long flash-back qui dévoile le terrible secret de la narratrice. Nos héroïnes ont découvert la vérité, Mary Alice nous la confie. Bien évidemment, nous voyons la véritable version, narrée par le personnage actant. Mary-Alice, transformée en mère meurtrière, nous apparaît alors elle aussi comme un mystère. La voix s'efface sous l'interrogation des images. Cette présence fantôme est donc une présence à la fois rassurante, parce que guide mais aussi trompeuse, puisque manipulatrice.

Mystère et empathie se mêlent dans une modulation étrange du point de vue. En pénétrant à l'intérieur des maisons, à l'intérieur des personnages, on dévoile une " noirceur abyssale ${ }^{12}$ ». La série est sans fond puisqu'elle bute toujours contre le mystère de l'empathie, c'est-àdire notre capacité à penser l'autre comme une intériorité sensible. le mystère empathique. Reste alors cette voix fantôme, ce personnage étrange au « petit ton à la fois amusé et mélancolique » qui surplombe cet étrange vivarium. Car finalement, la distance nécessaire qu'induit la voix off entraîne la série vers une auscultation des corps contemporains par des effets inattendus de représentation et de commentaire. La voix alors n'est plus récit, elle est bel et bien personnage et point de vue, elle est ad vitam commentaire post mortem .

\section{Commentaire et distanciation}

« La série navigue entre une distanciation consciente et une proximité immédiate avec les actions de ses personnages. En clair, elle plaque de l'affect sur sa propre mécanique ${ }^{13}$ ». Cette analyse pertinente d'Olivier Joyard décrit très bien le fonctionnement étrange de cette voix, de cette narration. Comme nous l'avons précédemment expliqué, nous sommes toujours avec et contre les personnages, entre empathie et mystère. Ainsi on voit bien, notamment à travers le premier épisode, comment la voix stigmatise chacune des héroïnes, la cantonne dans un rôle prédéfini. La voix qui nous montre tend finalement à nous représenter les choses. C'est le grand dilemme entre monstration et

\footnotetext{
${ }^{10} \mathrm{Au}$ regard de la série, on voit bien comment Mary-Alice guide totalement les épisodes. Sa disparition soudaine laisse place à un mystère de l'autre, son retour entraîne une forme d'empathie critique sur les personnages. Son entrée dans l'intime est toujours accompagné d'une distance critique, comme si le personnage obéissait à une mécanique des sentiments. ${ }^{11}$ Olivier Joyard in Les Inrockuptibles n ${ }^{\circ} 562$

${ }^{12}$ Olivier Joyard in Les Inrockuptibles $\mathrm{n}^{\circ} 562$

${ }^{13}$ Olivier Joyard in Les Inrockuptibles ${ }^{\circ}{ }^{\circ} 562$
} 
représentation. Montrer c'est pointer du doigt, c'est « attirer l'attention sur ", représenter induit une part de création, de mise en scène qui met en jeu la subjectivité de ce que l'on perçoit. Montrer n'ordonne pas un sens unique de lecture, représenter guide notre regard et suppose un sens à ce que l'on voit. On peut s'interroger sur le positionnement du point de vue dans un tel dispositif.

La construction narrative nous entraîne vers une dimension représentative des corps. La voix de Mary-Alice prend donc la forme d'un commentaire. Elle amène une distance sur les personnages qui nous permet de percevoir leur mécanique. La position surplombée qu'implique une voix post mortem crée une forme de jugement "divin ». Mary-Alice, libérée du poids d'être vivante, observe la mécanique de tous ses anciens voisins. Elle franchit les murs, les corps et perçoit en eux toute la tristesse, la déception, le malheur de cet enfer domestique. Chaque épisode est ainsi construit autour d'une trame thématique sourde qui se dévoile en tête et fin d'épisode. La série opère ainsi un mouvement du général au particulier pour une ouverture vers le général. A travers ce mouvement, c'est bien le fonctionnement narratif de la série qui se met en place. Le quotidien et le feuilleton se mélangent plus aisément.

Ainsi, dans l'ouverture, Mary-Alice évoque un comportement humain (la trahison, la défaite, l'envie, l'attente...) pour nous amener dans le quotidien d'une de nos hérö̈nes. La série se déroule, alternant les différentes strates de la fiction. Puis, dans un mouvement réunificateur, la voix reprend son allure surplombante et commente l'épisode. Tous les personnages, dans un grand travelling faussé ${ }^{14}$, sont réunis dans un même mouvement de pensée. Leurs quotidiens résonnent, le feuilleton y résonne de même. Tout rentre dans un grand mouvement sous le point de vue de la morte. Le spectateur, par ce final, repense alors la construction de l'épisode. Il perçoit soudainement la mécanique narrative, sans qu'il ait eu l'impression d'y être soumis. Le feuilleton et le quotidien se sont mêlés de manière aléatoire et soudain, dans les dernières minutes, ils forment un tout, unifié et cohérent, qui donne une pensée du monde. La voix démontre et démonte le comportement humain. Le feuilleton comme le quotidien est révélateur. Ainsi, si la voix est commentaire, morale, le récit est Fable. Mary-Alice, telle une entomologiste, tire des leçons ${ }^{15}$. La fiction devient révélatrice. On est donc dans une mise en récit qui, par le biais d'une narration complexe, tend à distancier le récit pour créer un effet de pensée. Le terme de distanciation, terme associé à une pratique théâtrale très précise, est à entendre hors d'un contexte politique

${ }^{14}$ Le travelling est faussé puisqu'il réunit dans un même mouvement différents espaces. Cet effet visuel renforce la volonté de cohérence.

15 «Marie Alice vient de lancer quelques flèches noires sur les images trop colorées, trop polies du bonheur domestique ». O. Joyard. Les Inrockuptibles $n^{\circ} 562$ 
précis. La distanciation dans Desperate Housewives passe par un art du décalage. La série exhibe sans cesse ses artifices de construction pour inviter le spectateur à une réflexion sur les mécanismes de création des personnages Pour autant, comme le souligne Olivier Joyard, la série entretient une proximité immédiate avec ses personnages. Tout en ayant un recul sur eux, une distance critique, elle n'exhibe pas des personnages antipathiques. Au contraire, comme on l'a vu, tout le sel de la série tient dans l'élaboration d'une fiction qui joue sur une empathie faussée, un mystère complice avec ses acteurs. O. Joyard parle avec raison d'affect dans la mécanique. Comme pour contredire les animaux mécaniques de Descartes, la série propose justement de montrer combien l'humain est une mécanique de sentiment. Le récit tend donc dans sa représentation à cumuler une double position du spectateur. Toujours conscient des enjeux de la fiction, de son cadre représentatif, il n'est pas moins touché par les personnages. Ici, la dialectique Brechtienne est presque complète même si les enjeux de la série sont moindres par rapport aux engagements du dramaturge. La série réussit à créer une fiction suffisamment fine et complexe, capable de cumuler différentes dynamiques de récit et de regard.

L'art du soap, art du rebondissement, de la fiction débridée trouve donc ici une matrice fictionnelle propice au renouveau. La fiction soap devient une dynamique de récit à laquelle correspond une dynamique de regard. Ainsi, à ce récit complexe et mouvant, s'ajoute un art de l'imagerie et de la référence visuelle où le mouvement des images construit un art du commentaire propre à la dimension critique de la série. Le quotidien feuilletonnesque devient un monde d'images et de souvenirs, où le passé plane toujours sur les gestes du monde. Le monde fictionnel devient alors un réseau de fiction antérieure que le spectateur réinvestit et dynamise.

\section{Codes et gestes : épuiser, répéter, créer}

La dimension feuilletonesque de la série est, comme on l'a vu, en étroite relation avec le soap opera. Ce format télévisuel comporte toute une série de codes et gestes canoniques, de figures de style, que le spectateur a assimilés. Lorsque l'on convoque l'art du soap, on fait appel à toute une galerie de personnages, de situations, de fictions. Desperate Housewives ne déroge pas à la règle. La série se construit bien autour d'un axe fictionnel propre au soap ${ }^{16}$. Mais elle fait appel aussi à toute une imagerie propre au code. Ainsi la représentation de Wisteria Lane est construite autour d'un art du décorum, propre au soap. Mais le terme « décorum » n'est pas innocent.

${ }^{16}$ Art du rebondissement, de la situation dramatique, croisement des personnages dans un univers restreint. 
Tout est trop propre dans cette banlieue. La série exhibe plus qu'elle ne montre. Il est toujours difficile de définir clairement une logique ostentatoire. La série joue, par un art du montage, avec un effet de répétition propre à décaler toute singularité. Les maisons se ressemblent, les femmes au foyer arborent le même sourire qui cache des dents carnassières. L'intérieur des maisons continue l'art du décorum. La série produit une image léchée, trop lisse et trop propre pour produire un effet de réalisme. Ainsi, comme le principe du commentaire-morale, le décor décale la monstration vers un effet de représentation. Le spectateur s'inclut donc dans un petit théâtre du quotidien fictionnel. Guidé par ce regard étrange entre empathie et distanciation, il va alors observer la logique de répétition de ces corps. Encore une fois, la dialectique brechtienne n'est pas incongrue. La série s'affirme bien comme une désarticulation des codes du soap. "Car la répétition est depuis le début la fondation de $D . H$. : le recyclage du soap tient justement sa finesse d'une propension à emprunter des chemins connus, voire rebattus pour en extraire conceptuellement une parole, une geste décisifs, un détail, le détail qui tue ${ }^{17} »$.

Il y a donc dans la série quelque chose de l'ordre du « found footage », procédé de vidéaste contemporain qui consiste dans le recyclage des figures reconnues et d'une désarticulation de leurs fonctionnements. La série emprunte des sentiers " rebattus ", c'est à dire des pistes fictionnelles attendues. Le soap ne se renouvelle pas tant à travers les histoires abordées mais bien par la manière de les aborder. La série est donc finalement une forme de méta-soap, une réflexion sur elle-même puisqu'elle tend à dégager des codes, des lois propres à son existence. Elle charrie avec elle tout un passé du soap, un passé de spectateur qu'elle réinvestit dans l'image. Pour mettre en place cette " gestus » de la fiction populaire, la série décale alors le corps de ces personnages en les transformant en image. Outre l'élégance suspecte, la propreté immaculée du quotidien, cette transformation s'opère en réduisant chacune à des identités strictes et concises. On voit bien, de manière superficielle, que chacune des femmes incarne une représentation possible de la Femme ${ }^{18}$. Mais au delà de cette caractérisation schématique, formant un éventail possible d'identification, on se rend compte que ce n'est pas tant à un quotidien personnel qu'à un passé fictionnel, que la série nous renvoie. Ainsi, les acteurs sont assimilés à un passif fictionnel, puisqu'ils proviennent tous de séries différentes (Les Feux de l'amour, Melrose Place, Côte Ouest...). Le spectateur perçoit les personnages à travers la palette de personnages que le corps de l'acteur a hébergée.

\footnotetext{
${ }_{17}$ Olivier Joyard in Les Inrockuptibles $\mathrm{n}^{\circ} 562$

${ }^{18}$ Bree : la femme parfaite frigide; Susan : la femme enfant gaffeuse ; Lynette : la mère débordée ; Gaby : la bombe sexuelle intéressée.
} 
Le suivi de la série fait appel à une mémoire collective de spectateur, un plaisir du récit dit classique, travaillé de l'intérieur par des éléments propres à la culture populaire pour créer de nouvelles formes. La fiction n'est donc plus première, elle s'incarne dans un grand livre de fictions puisque les corps ne sont plus innocents. Ainsi, le passif fictionnel des personnages nous amène à percevoir les effets de répétitions. Dans le corps passé se réincarne, se rejoue, des codes du soap. Pour exemple, Bree Van de Kamp qui apparaît comme une réincarnation, une version modernisée du personnage hystérique et diabolique que l'actrice Marcia Cross incarnait dans le soap des années 90 Melrose Place. Attention, cela ne signifie pas qu'il y ait un lien entre les deux personnages mais plutôt un effet palimpseste où le corps de l'acteur, son apparence, n'efface jamais complètement le corps qu'il a été. Ainsi, comment ne pas penser à Dale Cooper de Twin Peaks en voyant le nouveau mari de Bree, interprété par Kyle Maclachlan ? Le spectateur, presque inconsciemment, prolonge l'existence de personnages qu'il a connus dans ce nouveau terrain fictionnel du quotidien.

La série en forme d'album photo donne à voir les tics, la répétions des codes et des gestes des fictions. Rien est anodin à Wisteria Lane puisque tout découle de fictions antérieures, d'un passé des images. Séparations, rencontres, surprises, meurtres, trahisons, tout est reprise, tout est relecture analytique de la mécanique narrative. Les corps se répètent, les gestes se dévoilent dans leurs habitudes. Finalement, la série tend à se vider de son sens actanciel pour ne devenir qu'une "gestus », qu'un art de la figure et de la mimique. Mais, il ne faudrait pas figer le corps dans une répétition stérile. La série recycle et réemploie. On bascule alors de l'art du cliché à la figure iconique, du dire au voir.

\section{L'art du cliché : mouvement d'images vers le dévoilement.}

La question de la reprise, de la citation « clin d'œil » est un point épineux qui touche à des notions fluctuantes. On aurait tendance à ranger du côté de la « post-modernité » cinématographique l'art de la connivence, de la reconnaissance qu'implique l'utilisation de clichés. Pour autant, ce terme de "post-modernité » pose problème puisqu'il imposerait une négation du classicisme et de la modernité. La question n'est donc pas tant de savoir dans quel courant ranger la série mais bien plutôt d'étudier le fonctionnement et les racines de ces clichés. Si, comme on l'a vu, la série ne cesse de mettre en scène son propre fonctionnement, elle n'en fait pas moins appel à une mémoire cinéphilique de spectateur. Tout est passif fictionnel, tout est réemploi. Ainsi, la vision de la banlieue nous renvoie tout autant à la critique de Sam Mendes dans American Beauty qu'à la fantasmagorie 
d'Edward aux mains d'argent de Tim Burton. Le spectateur tisse un réseau de représentations passées, qu'il associe inconsciemment avec ce nouveau monde diégétique. Aux clichés du récit fait écho un sentiment de déjà-vu. On ne peut s'empêcher de tirer ces femmes aux foyers désespérées vers des hérö̈nes hollywoodiennes classiques. Si, comme on l'a vu, la série entretient des liens avec des fictions très contemporaines, elle prend racine dans ce terreau d'images canonisées qu'est le cinéma hollywoodien. Au vue de la série, on pense à trois influences majeures, trois œuvres matrices qui irriguent toute la série.

La première, la plus évidente c'est Women de George Cukor. L'art du commérage trouvait dans ce film toute sa quintessence. Le film de Cukor, comme la série, arrivait à faire basculer le quotidien parlé en une action trépidante. Dans Women, le microcosme féminin était secoué par un drame, l'abandon d'une femme qui allait ébranler par ricochet différents quotidiens. La série tire donc en partie sa construction de cette œuvre matrice et fondatrice dans l'émancipation de la représentation féminine à l'écran. Nos Women contemporaines s'affirment aujourd'hui « désespérées », mais les personnages de Cukor ne l'étaient-ils pas un peu? La ligne fictionnelle de la série est donc bien elle-même réemploi. Jacques Lourcelles dans son Dictionnaire du Cinéma évoque, lors d'une notice sur le film de Cukor, le style " parfaitement équilibré entre une certaine légèreté de touche et une sécheresse ironique » et souligne ainsi la dimension spectaculaire du film. On pourrait parfaitement apposer cette citation sur Desperate Housewives. Les deux œuvres ont en commun une capacité étonnante de mêler les personnages dans une valse hésitante du quotidien. Les péripéties s'emboîtent dans un mouvement empathique mais l'ironie n'est jamais loin. Il n'y a donc pas ici véritablement citation mais bien réemploi, superposition des deux œuvres. On retrouve encore ici, mais de manière détournée, cette logique du palimpseste qui vise, comme on le verra, par un processus d'accumulation, à créer une image constamment composite dont le spectateur devra intégrer la dynamique.

La mécanique narrative trouve aussi dans Chaînes Conjugales de Mankiewcz son œuvre clé. On y retrouve différents éléments décisifs : la banlieue américaine, la voix off d'une femme disparue qui crée un mystère (Addie Ross) et un coup de théâtre qui vient faire basculer le quotidien. Le film de Mankiewcz propose une construction aussi habile et étonnante que la série. La voix d'Addie, la voleuse de maris, s'efface et réapparaît entre les mailles des différents flash-backs qui construisent le film. Comme dans la série, nous basculons sans cesse entre intériorité et extériorité des personnages. Ici encore, l'esthétique de la banlieue hollywoodienne et la caractérisation typique des personnages apparaissent comme sous-jacentes à la série. Le suspense créé n'est pas étranger aux différentes interrogations que soulève constamment la série. Le drame domestique peut devenir un 
enjeu dramatique. Pour autant, c'est bien plutôt vers la troisième référence classique, peut-être la plus importante, qu'il faut aller puiser les situations cornéliennes et mélodramatiques, voire dérangeantes de la série : l'œuvre de Douglas Sirk.

Ses mélodrames irriguent encore le cinéma contemporain, de François Ozon à Todd Haynes. Mais c'est bien avec Desperate Housewives que l'œuvre de Sirk trouve son réemploi le plus abouti. En refondant dans une structure populaire les obsessions visuelles et thématiques de son œuvre, la série offre à Douglas Sirk une modernité confondante. Les héroïnes Sirkiennes, qu'elles soient interprétées par Janet Wyman ou Lauren Baccall, nous apparaissent bien comme les grands-mères de nos desperate housewives. Visuellement, la série emprunte ce goût du décor bourgeois, proche de l'artifice. Mais, comme l'a bien montré Sirk, l'artifice donne une consistance au monde. Les passions de ses personnages, leurs destins croisés, leurs erreurs, leurs ruptures font écho à celles de nos personnages contemporains. Le scénario du Secret Magnifique (1954), à travers ce croisement des destinées, est très proche de ce que la série pourrait produire comme type de fiction. Mais ce sont surtout les mélodrames domestiques comme Tout ce que le ciel permet (1955), Le mirage de la Vie (1959) et Ecrit sur du Vent (1956), auquel la série fait appel.

Dans ces trois films, on retrouve bien ce questionnement identitaire propre au franchissement de la frontière domestique. L'hystérie des personnages, leur débordement, fait écho à cette « banlieue modèle [...] où tout cela déborde : trop de pression trop de haine contenue ${ }^{19}$ ", que met en scène la série. La question de l'intériorité problématique est au cœur de l'œuvre de Sirk. Mais c'est surtout par des effets visuels forts que Sirk induit cette notion : on pense notamment à la multiplication des plans miroirs. Le cinéma de Sirk, comme la série, rentre telle la bourrasque inaugurale d'Ecrit sur du vent, dans les maisons, dans l'intimité de ses personnages pour en dévoiler la noirceur. Ecrit sur du Vent, de par sa rhétorique visuelle est sûrement l'œuvre la plus proche de Desperate Housewives. Par un art du rebondissement et de la péripétie mais aussi par des séquences visuelles fortes (la mort du patriarche ou le final), Sirk maintient une tension permanente sans jamais perdre cette distance étrange propre aux mélodrames.

On a souvent évoqué l'ironie sous-jacente de Sirk. Mais comme dans Desperate Housewives, la mise en image n'induit un décalage que pour mieux dévoiler le personnage. L'affect se loge dans ce hiatus de l'identification. Car finalement toutes ces œuvres n'utilisent pas, au sens strict, des clichés. Il y a dans le cliché une fixité incompatible avec toute la mécanique visuelle et narrative inhérente à ces œuvres. Comme le prouve le générique, le cliché se dynamise et

19 Olivier Joyard in Les Inrockuptibles $\mathrm{n}^{\circ} 562$ 
dévoile une vérité. Ce générique extrêmement symbolique joue sur les multiples représentations artistiques de la femme. Sur l'écran apparaissent des tableaux célèbres, depuis Eve jusqu'à l'héroïne bdéesque de Lichtenstein. La série assume donc son art du chromo, de la redite, en s'inscrivant presque dans une dimension muséale. Soudain, par le mouvement des images, le tableau s'anime et dévoile le cliché. Eve, la croqueuse de pomme, assomme Adam; le paysan typique américain cache une pin-up aux yeux de sa femme; la ménagère presque Warholienne fait déborder son sac de courses, etc. Ce générique est symptomatique du mouvement des images de la série. Le cliché s'anime et se dévoile par des effets de sens. Bree, Susan ou Lynette font sans cesse vaciller leur existence de papier glacé pour de nouveau s’incarner. Le cliché devient donc une dynamique de regard.

La reconnaissance tacite de son existence entraîne une surprise du décalage, de la dérive. Le personnage se décolle de son image, ou en révèle quelque chose. Le cliché les assimile à des catégories autres que moi ${ }^{20}$, mais soudain le dévoilement les ramène vers moi. Le personnage est donc sans cesse image et surplus de réel. Il y a dans ce décollement des apparences, ce hiatus de l'identification une force émotionnelle et d'attachement qui renforce le processus fictionnel. La mise en images crée une forme d'addiction du spectateur. La fiction, en se mettant en images, trouve alors son plus grand allié : le spectateur.

\section{Prolongement de la fiction}

La suspension de la fiction, son arrêt momentané, met le spectateur en crise. Au cœur de cette attente, il y crée sa propre fiction, une fiction potentielle ou prévisionnelle. L'épisode s'arrête mais le spectateur, lui, prévoit ce qui va se passer, il dénoue et renoue de luimême les fils du récit. C'est l'art du « petit malin » qui se croit plus fort que la fiction, en croyant la devancer. Tout le principe d'addiction tient ici dans le plaisir du « J'en étais sûr ! ». Le spectateur attend la suite pour vérifier ou non ces théories, pour valider ou non sa fiction potentielle. Elle se bâtit évidemment sur une reconnaissance de schémas, de figures clés de la fiction à travers la notion de genre. Le spectateur reproduit les mêmes histoires déjà vues, en superposant à la série toutes les autres fictions antérieures. La série se construit sur ce rapport complexe de reconnaissance et d'ironie. Le soap opera est investi comme tel, dans une reconnaissance tacite du cliché. Mais attention, comme le prouve le générique, le cliché s'anime et se dévoile par des effets de sens. C'est tout l'art de détruire les fictions

\footnotetext{
${ }^{20}$ Reconnaître un cliché c'est ne pas s’y intégrer. Etonnamment, le cliché a quelque chose de sociétal tant il est censé parler à tout le monde. La dynamique du cliché impose donc un regard sur sa propre société, et une forme d'abstraction de soi.
} 
prévisionnelles du spectateur. La reconnaissance de celui-ci, sa capacité à ranger une séquence dans une histoire des formes et des récits, c'est la création d'un espace dynamique entre lui et ce grand livre d'images. Chaque épisode produit ainsi son effet de prévision, de certitudes et d'évidences qui poussent le spectateur à la vérification. La modernité de la série tient dans cette conscience du cliché comme instrument d'accroche. La série cultive ainsi un goût pour l'image proche de l'imagerie ${ }^{21}$.

La force de Desperate Housewives réside dans ce melting pot complice d'images, cet amalgame tacite de références et d'icônes, cette fiction de toutes les fictions. La série produit un effet d'accumulation, où l'enchevêtrement des références, des personnages, des situations, des rebondissements, crée un effet de sur-place dynamique. Le spectateur pénètre dans la série et voit s'agiter autour de lui toutes les fictions d'antan. On voit très bien, notamment dans la saison 2, comment le personnage de Bree est transposé au cœur de multiples fictions, entre douleur du deuil (mélodrame), meurtres, trahison (suspense, thriller notamment par la figure du «stalker » George) puis drame familial et enfin film psychiatrique. Le personnage s'épuise d'ailleurs au cœur de cette multitude. L'assiduité du spectateur crée donc un effet « de trop plein » qui distord le processus fictionnel. En prolongeant la fiction, mais en sachant pertinemment que cette dernière n'est qu'une roue libre déchaînée, le spectateur s'insère dans un espace fictionnel impossible et donc forcément attrayant.

La question de la fiction suppose une suspension momentanée de l'incroyance. La série joue avec amusement de cette condition sine qua non. En ouvrant les vannes du possible, la série se débride et produit un quotidien extraordinaire et promet ainsi un renouveau permanent. Le spectateur dérive au cœur de ce grand tout fictionnel, guidé par les images qu'il reconnaît. "La signification de ce que je perçois surgit par le biais de la promesse ». Cette citation d'Albert Laffay démontre parfaitement combien, finalement, dans Desperate Housewives, la fiction s'articule sur un déroulement d'images. Voir, c'est tout d'abord percevoir, prévoir, envisager le déroulement. La série se réduit à un mouvement d'approche permanent. Le spectateur navigue au cœur des images vers un futur proche toujours renouvelable. La fiction dérive, se détourne, se retourne sur elle-même et emprunte tous les chemins qui mènent aux spectateurs. Renouveau permanent à travers une lecture d'images, assemblage de signes et de codes, la série s'iconise en permanence, en devenant son propre signifiant. Tout est réseau d'images et de sens, puisque tout est

${ }^{21}$ « Marc Cherry a plongé dans l'inconscient cinéphile de la télévision américaine. Le récit procède par strates, entrelacs, à la manière d'une conscience verrouillée par la névrose, qui laisserait tomber au compte-goutte les bribes d'une existence complètement tordue » $\mathrm{O}$. Joyard, Les Inrockuptibles $\mathrm{n}^{\circ} 578$. 
intentionnalité. En se vidant d'une structure univoque, en devenant toujours un futur possible, la fiction se vide de ses contingences et devient le cadre dans lequel s'anime le grand livre d'images.

Albert Laffay est connu pour avoir théorisé cette question de la monstration et de la représentation au cour de l'art cinématographique $^{22}$. Pour Laffay, il existe derrière les images, derrière le film « un grand Imagier », une structure qui « tournerait les pages et pointerait de son index » les éléments annonciateurs. Laffay crée une sorte de méga-narrateur, d'entité invisible qui souligne, donne à voir, sur l'écran, la promesse significative. La série se construit bel et bien autour de cette notion. De l'art de l'image, nous passons à ce grand Imagier qui guide le lecteur au cœur de cette fiction - cadre. A grands coups de crayon, les premiers épisodes dessinent les contours du cadre. Mais le tableau est mouvement, la fiction mute et se déplace sans cesse dans un renouveau permanent. Ce n'est que par cette structure du Grand Imagier que le spectateur perçoit les mouvements de la série.

La série se vide de son augure de récit continu pour devenir une page blanche sur laquelle s'impriment toutes les fictions. Chacune charrie avec elle ses stéréotypes, ses icônes et ses promesses. Au fil de saisons, les histoires se répètent, les gestes aussi. C'est par le suivi fictionnel que se dégage le véritable sens critique de la série. Ce processus d'accumulation vise finalement à dresser un panorama critique de la fiction américaine. En projetant au cœur de ce quotidien tous les fondements des fictions d'antan, la série en dévoile les tics, les répétitions, les insuffisances. Mais elle permet aussi un renouveau générique, un goût presque classique d'images cataloguées. Le spectateur, en emménageant à Wisteria Lane, s'insère au cœur d'un monde d'images et de fictions.

Mais, soudain, l'affect surprend et l'image se dévoile, se décale et la fiction-cadre permet un renouveau. Accumulations, répétitions, observations, la fiction n'est plus une ligne qui se trace mais un point central autour duquel tout part. Fiction de toutes les fictions, elle est rond-point à partir duquel le spectateur emprunte tous les chemins possibles de l'imaginaire qui fonde une Nation. On a ainsi souvent vanté la série pour ses vertus critiques. Puis très vite, les critiques se sont désolés d'un certain conservatisme. Mais ce n'est pas tant dans le propos politique de la série mais bien dans sa structure fictionnelle qu'il faut aller chercher le ferment analytique et critique. La série étale devant nos yeux l'imaginaire américain et en démonte le fonctionnement. La série s'affirme donc comme une proposition de «méta-fiction» feuilletonesque, qui juxtapose suivi distancié et identification empathique. Le spectateur fait l'expérience d'un récit qui sans cesse se défie de lui-même, et s'amuse à jouer avec ses propres contingences. Répéter pour mieux comprendre, rejouer pour mieux

${ }^{22}$ Albert Laffay, Logique du cinéma, Création et spectacle, Paris, Masson, 1964. 
penser semble être la devise de cette nouvelle structure fictionnelle qui privilégie l'art du collage, du melting pot à l'art rectiligne de la fiction rassurante.

Le suivi du spectateur est donc primordial au cœur de cette construction feuilletonesque. Le feuilleton se déploie par le regard analytique. Le quotidien, temps qui passe, devient temps de fiction. L'art du réemploi, du recyclage, tout en ne perdant pas sa dimension ironique, acquiert une justification émotionnelle. A l'image de son générique, la série se sait Histoire des images avant d'en être le renouveau. Henri Meschonnic explique, à juste titre, que la « modernité se choisit un passé »23. La série est donc éminemment moderne puisqu'elle est lien avec le passé, vers une dynamique du futur. Le renouveau de la série télévisée contemporaine américaine appelle donc à une lecture des œuvres passées. Pour comprendre ce qui se déroule sur l'écran, il ne faut pas s'abstraire d'une culture populaire des images. Dès lors, à la suite de Desperate Housewives, des séries comme Glee ou Chuck font de la culture pop le liant imaginaire entre la fiction et le spectateur.

\footnotetext{
${ }^{23}$ Henri Meschonnic, Modernité modernité, coll « Folio », Gallimard, 1994.
} 